\title{
Computing the density of states with the global Hybrid Monte Carlo
}

\section{Roberto Pellegrini*}

School of Physics and Astronomy, University of Edinburgh, Edinburgh EH9 3FD, UK E-mail:

r.pellegrinided.ac.uk

\section{Biagio Lucini}

College of Science, Swansea University, Singleton Park, Swansea SA2 8PP, UK

E-mail: b. lucini@swansea.ac.uk

\section{Antonio Rago}

Centre for Mathematical Sciences, Plymouth University, Plymouth, PLA 8AA, UK E-mail:

antonio.rago@plymouth.ac.uk

\section{Davide Vadacchino}

College of Science, Swansea University, Singleton Park, Swansea SA2 8PP, UK

E-mail: d.vadacchino@swansea.ac.uk

The LLR algorithm is a recent proposal for computing the density of states in lattice gauge theory. This algorithm has been tested in several bosonic models at zero and finite chemical potential with impressive results. Its original formulation is based on the simulation of the theory on restricted action intervals using local Monte Carlo updates. We will discuss a new version of the method based on the global Hybrid Monte Carlo algorithm that is suitable for theories with dynamical fermions. We shall present our preliminary result for the study of the SU(2) gauge theory.

34th annual International Symposium on Lattice Field Theory

24-30 July 2016

University of Southampton, UK

\footnotetext{
${ }^{*}$ Speaker.
} 


\section{The LLR algorithm}

Monte Carlo (MC) importance sampling simulations are one of the most powerful methods to study non-perturbative phenomena in quantum field theory. Despite their success, there is a number of known cases where they perform very poorly. For instance, in systems with a non-real action, the path integral measure is not positive-definite; as a consequence, to reach a given accuracy in a numerical computation using a direct implementation of importance sampling techniques, one would need a simulation time that scales exponentially with the size of the system. Another well known problem with exponential complexity is the computation of partition functions and free energies [1]; in fact these quantities cannot be expressed as expectation values over a probability measure and this leads to poor perfomances of MC importance sampling simulations.

An alternative method based on the density of states has been recently proposed [2, 3]. At action value $s$, the density of states $\rho(s)$ is defined via the functional integral

$$
\rho(s)=\int[D \phi] \delta(S[\phi]-s),
$$

where $\phi$ is some generic dynamical field variable, $\int D \phi$ is the path integral over the field configurations and $S[\phi]$ the action of the Euclidean quantum field theory. From the density of states it is possible to compute the partition function and expectation of observables via a simple one dimensional integration. For instance, if $O$ is a function of $s$,

$$
\langle O\rangle=\frac{\int \rho(s) O(s) e^{-\beta s} d s}{\int \rho(s) e^{-\beta s} d s}
$$

(see [3] for the case of a generic observable).

The LLR algorithm is a numerical method to compute the density of states from first principles that has proved to be very efficient in different models $[3,4,5,6]$ (see also the contributions $[7,8$, $9,10]$ to this conference). However, it relies on a simulation of the system in action intervals of given amplitude $\delta_{S}$. While in principle it is possible to simulate every system in a restricted action interval, it can be technically very difficult or inefficient. Here, we propose a new version of the LLR algorithm that is based on the global Hybrid Monte Carlo algorithm and avoids to constrain the system inside action intervals.

\section{Analytic support function}

The key idea of our method is to replace the sharp-cut in action of the LLR algorithm with a strongly localised analytic support function. The simplest possible choice is a Gaussian function. Following the lines of the original LLR algorithm, we define a double bracket expectation value by

$$
\langle\langle f(S)\rangle\rangle_{a, S_{0}, \sigma}=\frac{1}{\mathscr{Z}} \int f(S) \rho(S) e^{-a S} e^{-\frac{\left(S-S_{0}\right)^{2}}{2 \sigma^{2}}} d S,
$$

where

$$
\mathscr{Z}=\int \rho(S) e^{-a S} e^{-\frac{\left(S-S_{0}\right)^{2}}{\sigma^{2}}} d S .
$$


and $a$ is a parameter. Considering the simple observable $\left(S-S_{0}\right)$, we have

$$
\left\langle\left\langle S-S_{0}\right\rangle\right\rangle_{a, S_{0}, \sigma}=\frac{1}{\mathscr{Z}} \int\left(S-S_{0}\right) \rho(S) e^{-a S} e^{-\frac{\left(S-S_{0}\right)^{2}}{2 \sigma^{2}}} d S .
$$

We Fourier-transform the Gaussian function, obtaining

$$
\left\langle\left\langle S-S_{0}\right\rangle\right\rangle_{a, S_{0}, \sigma}=\frac{\int\left(S-S_{0}\right) \rho(S) e^{-a S} \int d k e^{i k S_{0}} e^{-\sigma^{2} k^{2}} d S}{\int \rho(S) e^{-a S} \int d k e^{i k S_{0}} e^{-\sigma^{2} k^{2}} d S} .
$$

For small $\sigma$, we can expand both numerator and denominator in powers of $\sigma^{2}$, which gives

$$
\left\langle\left\langle S-S_{0}\right\rangle\right\rangle_{a, S_{0}, \sigma}=\frac{\sigma^{2}}{2} \frac{\left.\frac{d \rho}{d S}\right|_{S_{0}}-a \rho\left(S_{0}\right)}{\rho\left(S_{0}\right) e^{-a S_{0}}}+\mathscr{O}\left(\sigma^{4}\right) .
$$

If we impose that $\left\langle\left\langle S-S_{0}\right\rangle\right\rangle_{a, S_{0}, \sigma}=0$, we obtain

$$
\left\langle\left\langle S-S_{0}\right\rangle\right\rangle_{a, S_{0}, \sigma}=0 \Longrightarrow a=\left.\frac{d \log \rho}{d S}\right|_{S_{0}} .
$$

By virtue of the above equation, in the same spirit of the original LLR method, the problem of computing the density of states is reduced to the problem of finding the coefficients $a_{k}$ such that the double brackets expectation values in eq.(2.5) are zero. From the $a_{k}$, an approximate density of states that converges to the real one in the limit $\sigma \rightarrow 0$ can be reconstructed imposing piece-wise continuity of $\rho$. This yields

$$
\rho(S)=\rho_{0}\left(\prod_{k=1}^{N-1} e^{a_{k} \sigma}\right) e^{a_{N}\left(S-S_{N}\right)} .
$$

In order to compute the double brackets expectation values in eq.(2.5) we will need a Markov Chain Monte Carlo (MCMC) that will sample the configurations with weight given by

$$
W\left(S[\phi], S_{0}, \sigma, a\right) \propto e^{-a S} e^{-\frac{\left(S-S_{0}\right)^{2}}{2 \sigma^{2}}}=e^{-U\left[\phi, S_{0}, \sigma, a\right]} .
$$

A natural approach is to use a global Hybrid Monte Carlo.

\section{The global Hybrid Monte Carlo}

The first step for defining a global Hybrid Monte Carlo (HMC) sampler is a differentiable potential function. In our case the function

$$
U\left[\phi, S_{0}, \sigma, a\right]=a S+\frac{\left(S-S_{0}\right)^{2}}{2 \sigma^{2}}
$$

in eq.(2.8) can be used as the potential energy of the HMC sampler. The next step is to define the canonical conjugate field $p_{i}$ and add to the Hamiltonian the kinetic term, which gives

$$
H\left[p_{i}, \phi_{i}\right]=\sum_{i} \frac{p_{i} p_{i}}{2}+U\left[\phi, S_{0}, \sigma, a\right] .
$$


At this point we are able to define the force as the gradient of the Hamiltonian, i.e.

$$
f_{i}=-\frac{\partial H}{\partial \phi_{i}}=-\frac{\partial S}{\partial \phi_{i}}\left(a+\frac{1}{\sigma^{2}}\left(S-S_{0}\right)\right) .
$$

This formula shows that the molecular dynamic force in our case is proportional to the one in the standard HMC, with the proportionality factor being straightforward to compute.

The Hamiltonian evolution is given by the usual Hamilton equation and can be solved numerically by using any volume preserving integrator algorithm. At the end of the Hamiltonian evolution the the configuration is accepted or rejected according to the usual Metropolis test, with acceptance that can be tuned to an optimal value by changing the integration step.

Let us remark that due to the term $\frac{1}{\sigma^{2}}\left(S-S_{0}\right)$ the forces are very large during thermalisation, while they become much smaller once the action of the system is close to the center of the Gaussian $S_{0}$. To overcome the problem of large forces, during thermalisation we change the integration step after every Metropolis test in order to keep the acceptance ratio close to 0.8 .

We are now in a position to compute by MCMC sampling the double brackets expectation values in eq.(2.5). However our final goal is to solve the stochastic equation

$$
\left\langle\left\langle S-S_{0}\right\rangle\right\rangle_{a, S_{0}, \sigma}=\Delta S(a)=0 .
$$

The stochastic nature of the latter is due to the fact that we cannot have an exact determination of the function $\Delta S(a)$, which is known only through Monte Carlo estimates. The numerical solution of stochastic equations can be found using the iterative Robbins-Monro procedure [11]. This begins with a guessed value $a_{0}$ which is updated using the iteration

$$
a_{n+1}=a_{n}-c \frac{\left\langle\left\langle S-S_{0}\right\rangle\right\rangle}{n},
$$

see [3] for more details. Once the $a$ 's are computed the density of states is reconstructed using eq.(1.1), with errorbars determined using bootstrap resampling.

\section{Replica exchange method}

In principle, the algorithm proposed in the previous section is ergodic: given enough time, the algorithm will explore the entire phase space. On the other hand, the probability to visit states with action very far from the peak of the Gaussian function will be very small and this may lead to a slow dynamic of the Markov Chain. In order to overcome this problem, the method can be easily combined with replica exchange steps (see e.g. [12] for a similar approach in the Wang-Landau case). The idea is to simulate multiple intervals in parallel and periodically propose a swap of two of them with probability given by

$$
P_{s w}=\min \left(1, e^{U\left(a, S_{0}\right)+U^{\prime}\left(a^{\prime}, S_{0}^{\prime}\right)-U\left(a^{\prime}, S_{0}^{\prime}\right)+U^{\prime}\left(a, S_{0}\right)}\right),
$$

where $a, S_{0}, U$ refers to the first system and $a^{\prime}, S_{0}^{\prime}, U^{\prime}$ to the second one. The function $U$ is defined in eq.(3.1). In fig. (1) we report an example of $S U(2)$ gauge theory simulated using this technique. Swaps of replica are frequent, determining a faster evolution of the Markov Chain than in the case of sampling over single intervals. 


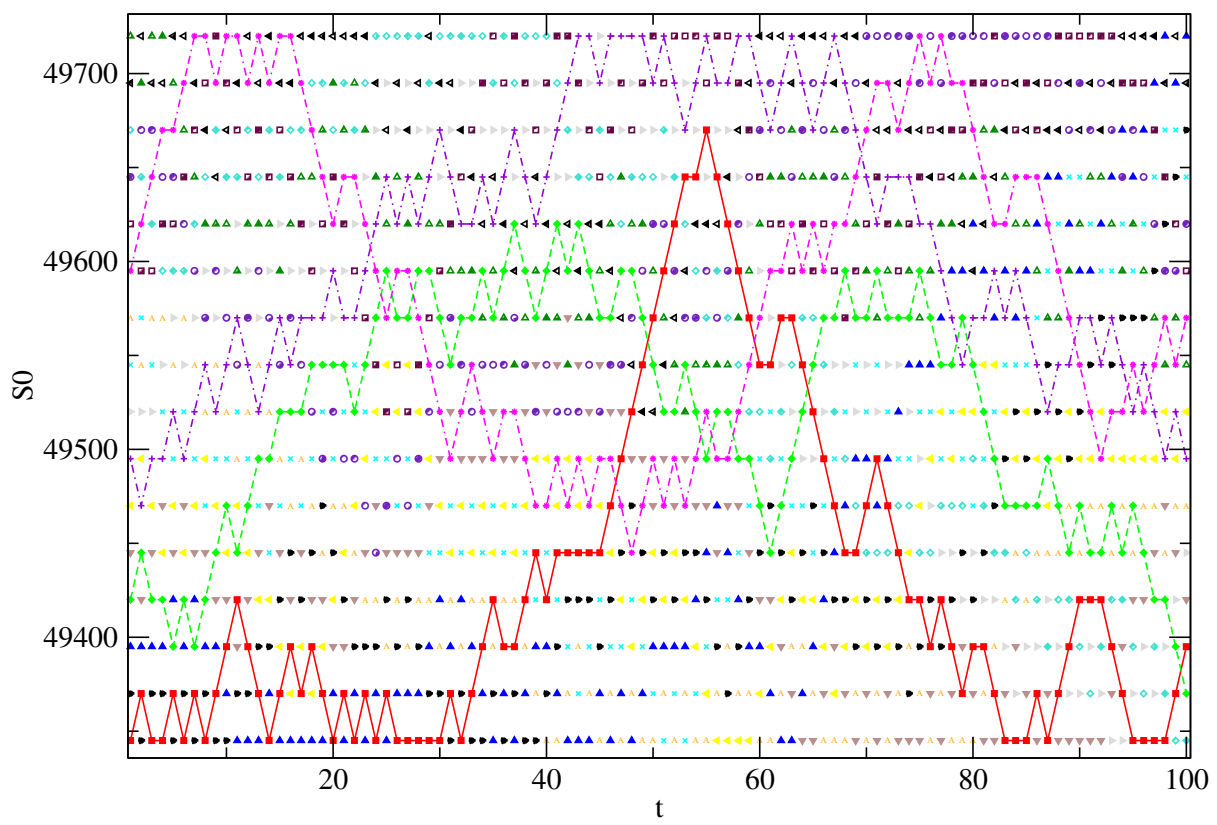

Figure 1: In this figure we plot the MC histories of 16 replicas for a $16^{3} \times 3$ lattice. Different symbols correspond to different replicas and we connected the symbols of four selected replicas to guide the eyes. The y-axis shows the peak of the Gaussians, while on the $\mathrm{x}$-axis we report the MC time. It is clear that with the chosen set of parameters the swap probability is fairly high.

\section{Application to the energy momentum tensor in Yang-Mills field theory}

The energy momentum tensor is a fundamental quantity in quantum field theory that provides the set of conserved Noether currents originating from translational invariance. In Yang-Mills theory it is possible to show that if the theory is regularised in such a way that translational symmetry is preserved, the energy momentum tensor does not need renormalisation and is given by

$$
T_{\mu v}=\frac{1}{g^{2}}\left\{F_{\mu \alpha} F_{v \alpha}-\frac{1}{4} \delta_{\mu v} F_{\alpha \beta} F_{\alpha \beta}\right\} .
$$

When using lattice regularisation, translational symmetry is explicitly broken and the energy momentum tensor needs to be renormalised [13]. The renormalised energy momentum tensor can be written as the sum of three operators,

$$
T_{R \mu \nu}=Z_{T}\left\{T_{\mu \nu}^{[1]}+z_{t} T_{\mu \nu}^{[3]}+z_{s}\left(T_{\mu \nu}^{[2]}-\left\langle T_{\mu \nu}^{[2]}\right\rangle\right)\right\}
$$


where the three operators are

$$
\begin{gathered}
T_{\mu \nu}^{[1]}=\left(1-\delta_{\mu \nu}\right) \frac{1}{g_{0}^{2}}\left\{F_{\mu \alpha}^{a} F_{v \alpha}^{a}\right\}, \\
T_{\mu \nu}^{[2]}=\delta_{\mu \nu} \frac{1}{4 g_{0}^{2}}\left\{F_{\alpha \beta}^{a} F_{\alpha \beta}^{a}\right\}, \\
T_{\mu \nu}^{[3]}=\delta_{\mu \nu} \frac{1}{g_{0}^{2}}\left\{F_{\mu \alpha}^{a} F_{\mu \alpha}^{a} F_{\alpha \beta}^{a} F_{\alpha \beta}^{a}\right\} .
\end{gathered}
$$

Using shifted boundary conditions [14] for the fields in the time direction

$$
A\left(L_{0}, x\right)=A\left(0, x-L_{0} \xi\right)
$$

it is possible to write a set of Ward Identities [15] that fixes the renormalisation constants $Z_{T}, z_{t}, z_{s}$. Here we focus on the renormalisation constant $Z_{T}$ which can be obtained from the formula

$$
Z_{T}(\beta)=\frac{f\left(\beta, L_{0}, \xi-a \hat{k} L_{0}\right)-f\left(\beta, L_{0}, \xi+a \hat{k} L_{0}\right)}{2 a} \frac{1}{\left\langle T_{0 k}^{[1]}(\beta)\right\rangle_{\xi}},
$$

with $f\left(\beta, L_{0}, \xi\right)$ the free energy of the system with shift $\xi$, coupling $\beta$ and inverse temperature $L_{0}$. The free energy can be computed from the density of states:

$$
f\left(\beta, L_{0}, \xi\right)=\frac{\log \int d S e^{(-\beta S)} \rho(S)}{V}+c .
$$

In the left panel of fig. (2) we plot the density of states as a function of the action for two $12^{3} \times$ 3 lattices with shifts $\left(\frac{4}{3}, 0,0\right)$ and $\left(\frac{2}{3}, 0,0\right)$ respectively. We would like to remark that we can appreciate the small difference between the two densities of states for a large range of orders of magnitude. The right panel of fig. (2) is the probability density for the same lattices at $\beta=2.36869$, and again we are able to measure the small difference between the two distributions even when the probability density is extremely suppressed with respect to the peak. With the same parameters we computed the difference of free energy by changing the shift from $\left(\frac{4}{3}, 0,0\right)$ to $\left(\frac{2}{3}, 0,0\right)$, obtaining percent precision on our value,

$$
\Delta f(\beta=2.36869)=0.002319(21) .
$$

\section{Conclusions}

In this contribution we presented a new version of the LLR algorithm to compute the density of states that is based on the Hybrid Monte Carlo algorithm. The main advantages of this new formulation is that it is very general and can be used for every continuos model for which it is possible to compute the gradient of the action. In addition, it avoids to constrain the system in action intervals, which can be technically difficult to implement.

In principle, the extension of our method to theories with fermionic degrees of freedom is straightforward. We are currently testing the algorithm for the computation of the energy momentum tensor in $S U(2)$ Yang-Mills theory and our preliminary results seem very encouraging. 

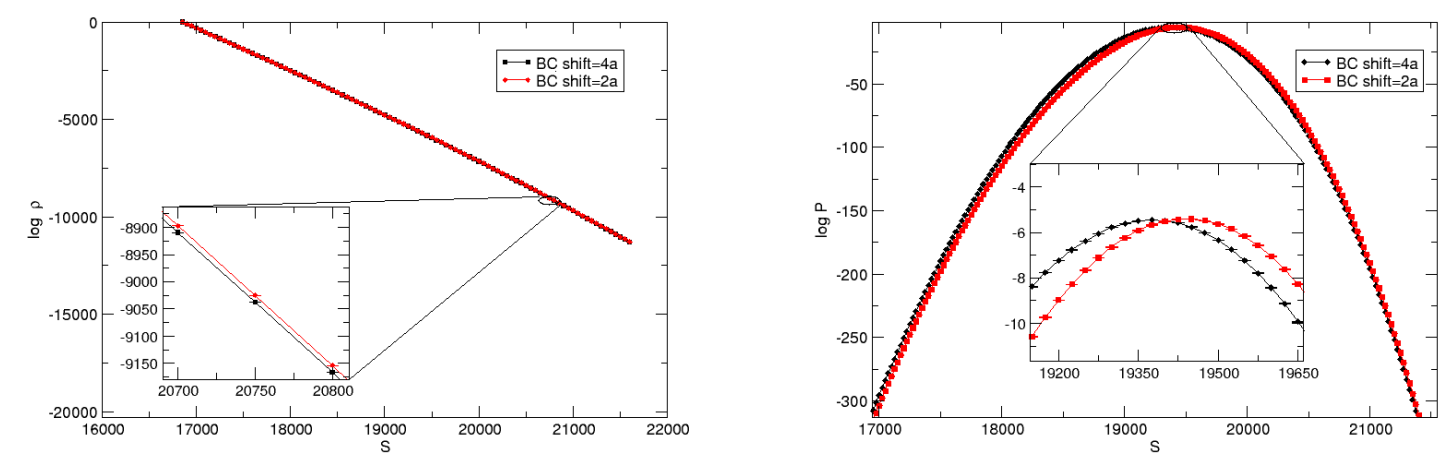

Figure 2: Left: density of states for vol $=12^{3} \times 3$ and shifts $\xi=\left(\frac{4}{3}, 0,0\right)$ and $\xi=\left(\frac{2}{3}, 0,0\right)$. Right: Probability density for $\beta=2.36869$, vol $=12^{3} \times 3$ and shifts $\xi=\left(\frac{4}{3}, 0,0\right)$ and $\xi=\left(\frac{2}{3}, 0,0\right)$.

\section{Acknowledgments}

The work of BL and DV is partially supported by the STFC Consolidated Grant ST/L000369/1. AR is supported by the Leverhulme Trust (Grant RPG-2014-118) and STFC (Grant ST/L000350/1). RP is supported by STFC (Grant ST/L000458/1). The numerical computations have been carried out using resources from the HPCC Plymouth and the HPC Wales (supported by the ERDF through the WEFO, which is part of the Welsh Government).

\section{References}

[1] P. de Forcrand, M. D’Elia, and M. Pepe, Phys.Rev.Lett.86, 1438 (2001).

[2] K. Langfeld, B. Lucini and A. Rago Phys. Rev. Lett. 109 (2012).

[3] K. Langfeld, B. Lucini, R. Pellegrini and A. Rago arXiv:1509.08391 (2015).

[4] K. Langfeld, B. Lucini Phys. Rev. D 90, 094502 (2014).

[5] K. Langfeld, J. Pawlowski Phys. Rev. D 88, 071502(R) (2013).

[6] C. Gattringer, P. Torek Phys. Lett. B Vol. 747 (2015).

[7] K. Langfeld, arXiv:1610.09856 [hep-lat].

[8] B. Lucini, W. Fall and K. Langfeld, arXiv:1611.00019 [hep-lat].

[9] N. Garron and K. Langfeld, arXiv:1611.01378 [hep-lat].

[10] M. Giuliani, PoS LATTICE 2016, 040 (2016) [arXiv:1611.03719 [hep-lat]].

[11] H. Robbins, S. Monro Ann. Math. Statist. Volume 22, Number 3 (1951).

[12] Thomas Vogel, Ying Wai Li, Thomas Wust, David P. Landau Phys. Rev. Lett. 110, 210603 (2013).

[13] S. Caracciolo et al., Nuclear Phys. B Vol 9 (1989).

[14] L. Giusti, H. B. Meyer Phys. Rev. Lett. 106 (2011).

[15] L. Giusti, M. Pepe, Phys. Rev. D 91 (2015). 